\title{
Keratomycosis due to Paecilomyces lilacinus: A Case Report
}

\author{
Kalpana Suresh \\ Kalpana Suresh, MS, FICO, FRCS(Glasg), FLVPEI, Professor and Head of the Department of Ohthalmology, \\ Sri Ramachandra Medical College and Research Institute, Sri Ramachandra University, Porur, Chennai, India-600116
}

We report here a case of keratomycosis by Paecilomyces lilacinus. 57-year-old farmer presented to us with pain, watering and defective vision in his left eye of 2 weeks duration. He gave history of injury to left eye with wooden piece. He was admitted and treated elsewhere, with no relief of symptoms. He was not a known diabetic. Other medical history was not relevant.

On examination of his left eye, there was dense stromal infiltrate measuring $6 \times 5 \mathrm{~mm}$ in the inferior cornea from 5 to 7'o clock hours, extending onto inferior pupillary margin (Figure 1). There was no lucid interval. Margins were ill defined. Cornea surrounding the ulcer was hazy due to stromal edema. Anterior chamber had $3 \mathrm{~mm}$ hypopyon admixed with blood with grade 4 reaction. Pupil was $3 \mathrm{~mm}$ reacting to light. Fundus view was hazy due to corneal infiltrate. His best-corrected visual acuity was finger counting at $3 \mathrm{mts}$. Right eye examination was within normal limits.

Labs: Grams stain showed no organisms. $\mathrm{KOH}$ wet mount showed fungal filaments. Blood sugar was $98 \mathrm{mg} \%$ Liver function tests were within normal limits.

Patient received topical Natamycin 5\% suspension one hourly, cycloplegic and oral anti-inflammatory drugs. Culture on SDA yielded no growth. The patient did not respond to the above therapy. Worsening of symptoms and signs noted along with impending perforation.

Therefore therapeutic keratoplasty was performed which was slightly decentered inferiorly (Figure 2). The corneal button was sent for microbiological and histopathological examinations. Post operatively the patient was treated with topical Natamycin 5\%, topical Amphotericin B 0.15\% and oral fluconazole. The patient did not show any improvement.

\section{Address for correspondence:}

E-mail: kalpanasrao@hotmail.com

DOI: 10.5530/ijmedph.3.2011.14
There was recurrence of infection in the graft. Progression resulted in perforation of the corneal graft, which required sclerocorneal re-graft (Figure 3). The patient was subsequently discharged. On follow-up the graft was taken up and the eye was quiet.

Microbiology studies yielded growth. The colonies were initially white, velvety and powdery (Figure 4) which turned into buff coloured later (Figure 5). Lactophenol cotton blue stain of the smear showed the presence of septate hyphae with long tapering phialides and globose conidia (Figure 6). This picture was suggestive of Paecilomyces Lilacinus.

Human mycotic keratitis is caused by filamentous, yeast or dimorphic fungi. More than 70 genera of moulds and yeasts have been associated with keratomycosis ${ }^{1}$. Filamentous fungi can further be classified as septate or nonseptate groups. Septate group includes pigmented and nonpigmented forms. Non-pigmented species includes aspergillus, fusarium and paecilomyces.

Paecilomyces are inhabitants of soil, decaying plants, food products and insects. There are two subspecies, namely Paecilomyces Lilacinus and Paecilomyces Variotii. They are thermophilic fungi and grow even at $50^{\circ}$ or $60^{\circ} \mathrm{C}$. The colony growth is initially velvety and white, becoming yellow and brown later. The colonies have sweet aromatic odour. They possess phialides, which are long, delicate and tapering along with globose conidia. Normally they grow within 3 to 4 days. However, 25\% take 2 to 3 weeks to grow.

Ocular involvement in the form of keratitis or endophthalmitis, occurs in immunocompromised persons, contact lens users or following intraocular surgery. The organism is resistant to natamycin, amphotericin B and 5 flurocytosine. It has variable sensitivity to imidazoles - ketoconazole, miconazole and econazole ${ }^{2}$. Newer agents like voriconazole and terbinafine are reported to be effective ${ }^{3}$. However, medical management is not effective in many patients. Most of the reports say that surgery is 


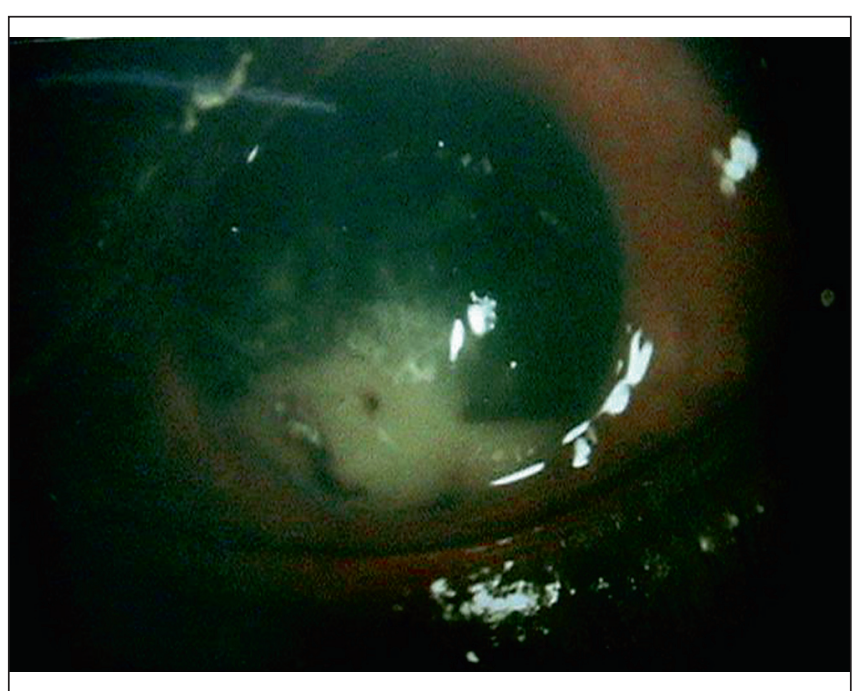

Figure 1

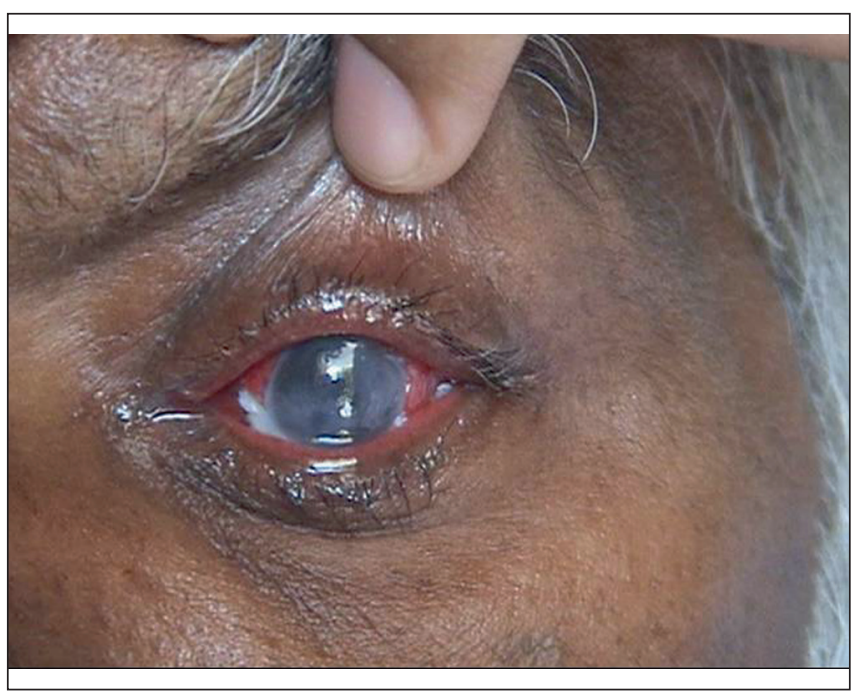

Figure 3

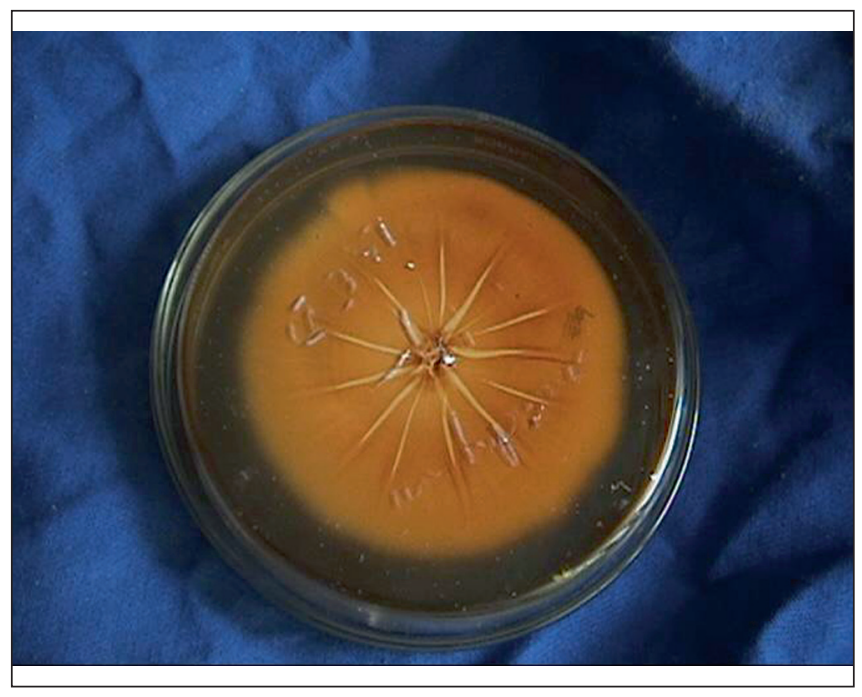

Figure 5

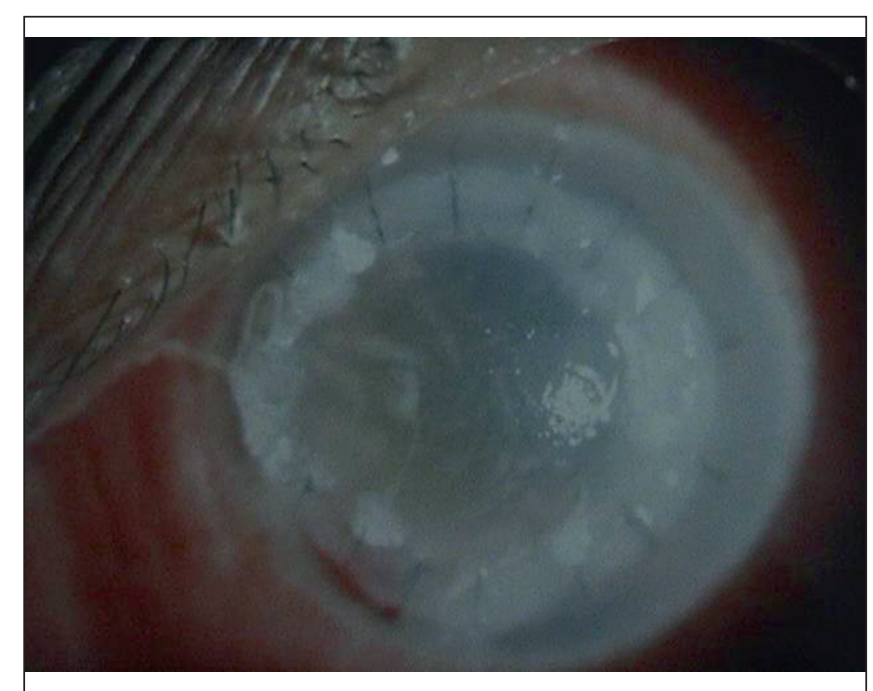

Figure 2

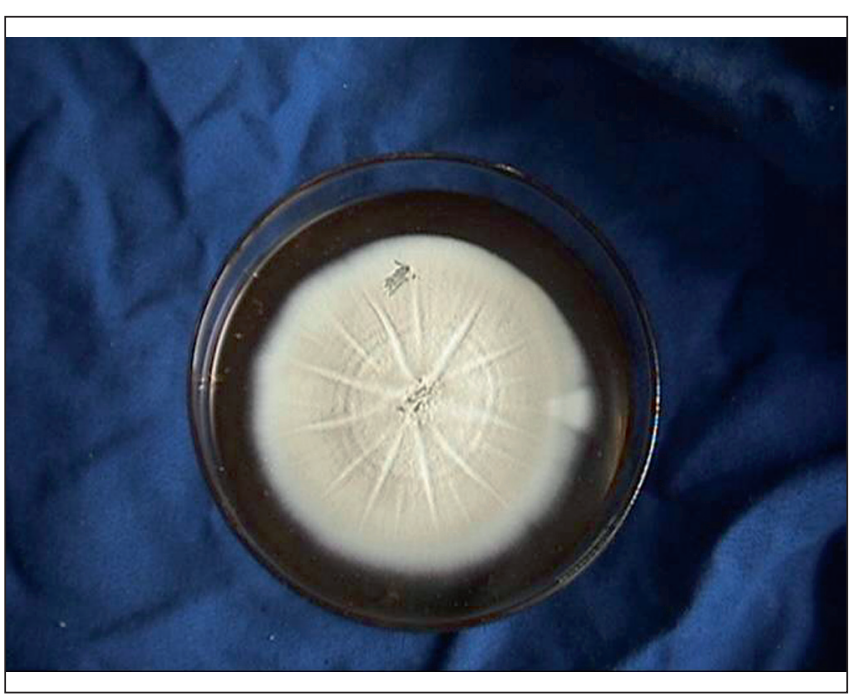

Figure 4

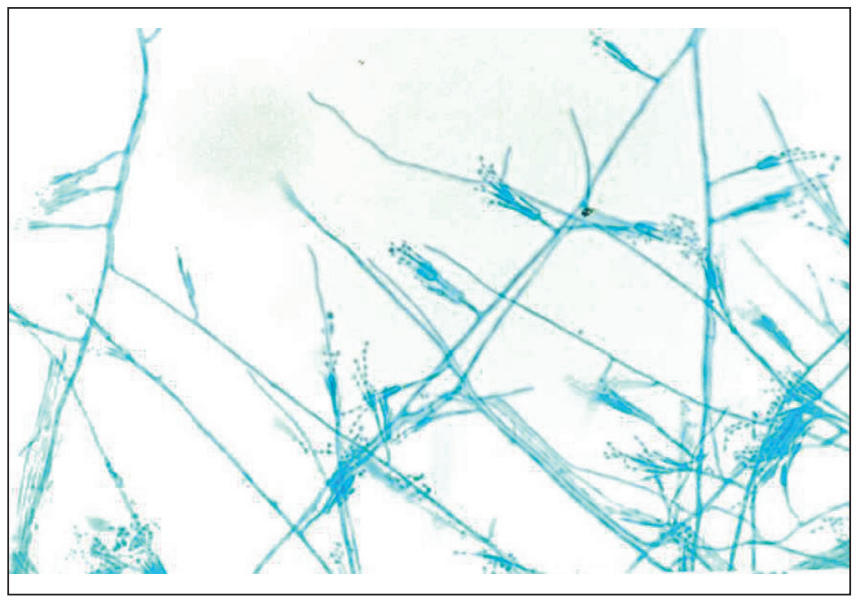

Figure 6 
required ultimately in most of them. The type of surgery includes penetrating keratoplasty, sclerocorneal graft or evisceration.

Late presentation, severe deep keratitis, delayed growth in culture and poor response to medical therapy was responsible for unsatisfactory outcome in this patient. Early recognition and medical management along with or without surgical intervention should be made in a case of paecilomyces keratitis.

\section{REFERENCES}

1. Prajna NV, Rao RA, Mathen MM, Prajna L, GeorgeC, Srinivasan M Simultaneous bilateral Fungal Keratitis caused by different fungi. Indian JOpthalmol 2002; 50:213-214.

2. Ana Espinel-Ingroff. In vitro activities of voriconazole, itraconazole and amphotericin B against opportunistic moniliaceous and dematatiaceous fungi. Journal of clinical microbiology, Mar 2001; 954-958

3. Garbino J, Ondrusova A, Baligvo E, et al. Successful treatment of Paecilomyces lilacinus endophthalmitis with voriconazole. Scand J Infect Dis $2002 ; 34: 701-703$ 